CLINICAL REPORT

\author{
E. Tollard \\ L. Niemtschik \\ T.E. Darsaut \\ F. Guilbert \\ D. Roy \\ J. Raymond \\ A. Weill
}

\title{
Endovascular Parent Artery Occlusion for the Treatment of Wide-Neck A1 Segment Aneurysms: A Single-Center Experience
}

SUMmARY: The goal of this study was to evaluate the feasibility and efficacy of A1 occlusion at the level of wide necked $A 1$ aneurysms, where there are bilateral patent $A 1$ segments and a patent AcomA. Between 2000 and 2010, 9 patients with wide necked A1 aneurysms were treated by coiling of the aneurysm along with parent vessel occlusion. All aneurysms had a wide neck $(\geq 4 \mathrm{~mm})$. None were treated in the acute phase of a subarachnoid hemorrhage. Three small infarcts were noted on routine post-treatment head CT, 1 of which was symptomatic (transient hemiparesthesia). On control angiogram at 6 months or more, 3 A1 recanalizations were found, 2 of which had a stable small neck recurrence. None of the aneurysms ruptured on follow-up. In this series, parent artery occlusion was effective in treating wide-necked aneurysms arising from the A1 segment in patients with adequate collateral supply.

\begin{abstract}
ABBREVIATIONS: $A 1=$ the first segment of the $A C A$ up to the $A$ comA; $A 2=$ the second segment of the ACA from the AcomA to the genu of the corpus callosum; $A C A=$ anterior cerebral artery; AcomA $=$ anterior communicating artery; $c=$ coiling; Dist $=$ distal third of the $A 1$; DSA = digital subtraction angiography; $E V T=$ endovascular treatment; $F U=$ follow-up; $L=$ left; $M D=$ maximum diameter; $\mathrm{Mid}=$ middle third of the $\mathrm{A} 1 ; \mathrm{MRA}=\mathrm{MR}$ angiography; $\mathrm{N}=$ neck; $\mathrm{OD}=$ oculi dexter (right eye); Prox $=$ proximal third of the $A 1 ; R=$ right; $R A H=$ recurrent artery of Heubner; $s=$ surgery; $\mathrm{SAH}=$ subarachnoid hemorrhage
\end{abstract}

$\mathbf{P}$ roximal ACA (A1) aneurysms are rare, accounting for $0.8 \%-3.4 \%$ of intracranial aneurysms. ${ }^{1-3}$ Wide-neck aneurysms, not only those located at A1, can be challenging to treat selectively by using an endovascular approach, and recurrences are more frequent even if treatment is successful. ${ }^{4}$ When the patient has adequate collateral vessel pathways (a patent AcomA with an A1 segment on both sides), occlusion of the A1 segment at the level of the aneurysm may be considered.

\section{Materials and Methods}

We studied all patients with ACA aneurysms included in our data base and treated by endovascular methods during a 10-year period (January 2000 to January 2010). From this list, all patients treated for an A1 aneurysm by using parent vessel occlusion were selected for a systematic medical record and imaging review.

The decision to occlude the parent vessel was always an a priori decision and was made after demonstration of collateral supply of the ACA by the contralateral carotid artery, by using manual compression or balloon-test occlusion. The parent vessel occlusion was never the result of a periprocedural complication.

Our strategy consisted of coiling the aneurysm tightly and letting the coils bulge through the neck. Parent vessel occlusion was completed with coils when necessary, with the intention of keeping the occlusion as short as possible. All the procedures were performed with the patient under heparin administration to reach 3 times the baseline of anticoagulation time.

Received March 29, 2010; accepted after revision May 31.

From the Department of Neuroradiology (E.T., L.N., T.E.D., F.G., D.R., J.R., A.W.), Centre Hospitalier de I'Universite de Montreal, Montreal, Canada; Department of Radiology (E.T.), University Hospital of Rouen, Rouen, France; and Department of Radiology (L.N.), Hospital Vargas, Caracas, Venezuela.

Please address correspondence to Eleonore Tollard, MD, University Hospital of Rouen, Radiology, 1 rue de Germont, Rouen, 76031, France; e-mail: eleonore.tollard@chu-rouen.fr DOI 10.3174/ajnr.A2222
With time, our policy regarding antiplatelet therapy changed. We are now administering $325 \mathrm{mg}$ of aspirin the day prior to treatment of unruptured aneurysms. In case of parent vessel occlusion, we continue the aspirin regimen for 3 weeks.

Follow-up neurologic examination (bedside sensitivomotor examination, including visual field evaluation) was performed by the interventional neuroradiologist daily before discharge and at 2 months. A CT scan was systematically obtained 24 hours after embolization to exclude asymptomatic complications. For all patients, a DSA or an MRA was performed 5-10 months after treatment. For patients with long-term imaging follow-up, DSA or MRA was performed 1-9 years after treatment.

\section{Illustrative Cases}

Case 1. A 55-year-old woman (patient 5) with breast cancer underwent a brain MR imaging performed to rule out metastases. A wide-neck aneurysm (5-mm neck, 10-mm diameter) (Fig 1) was found on the proximal third of the left A1 segment. After surgical resection of a low-grade breast cancer, the intracranial aneurysm, along with the parent vessel, was treated by occlusion with platinum coils, after verifying the patency of the AcomA and contralateral A1 segment by carotid compression. The postoperative course and CT findings were unremarkable. Follow-up DSA at 3 months and MRA at 2.5 years demonstrated complete occlusion of the aneurysm and A1.

Case 2. A 59-year-old woman (patient 7) underwent MR imaging of the brain for tinnitus. MR imaging revealed an incidental wideneck aneurysm (5-mm neck and 9-mm maximum diameter) (Fig 2) located on the distal third of the right Al segment. The EVT consisted of primary occlusion of the aneurysm, along with the parent vessel, with platinum coils after verifying the patency of the AcomA and contralateral A1 segment by right carotid compression while injecting the left internal carotid artery. A 24-hour CT demonstrated a small infarct of the right anterior pallidum and the genu of the internal capsule; however, the patient remained asymptomatic. Follow-up 

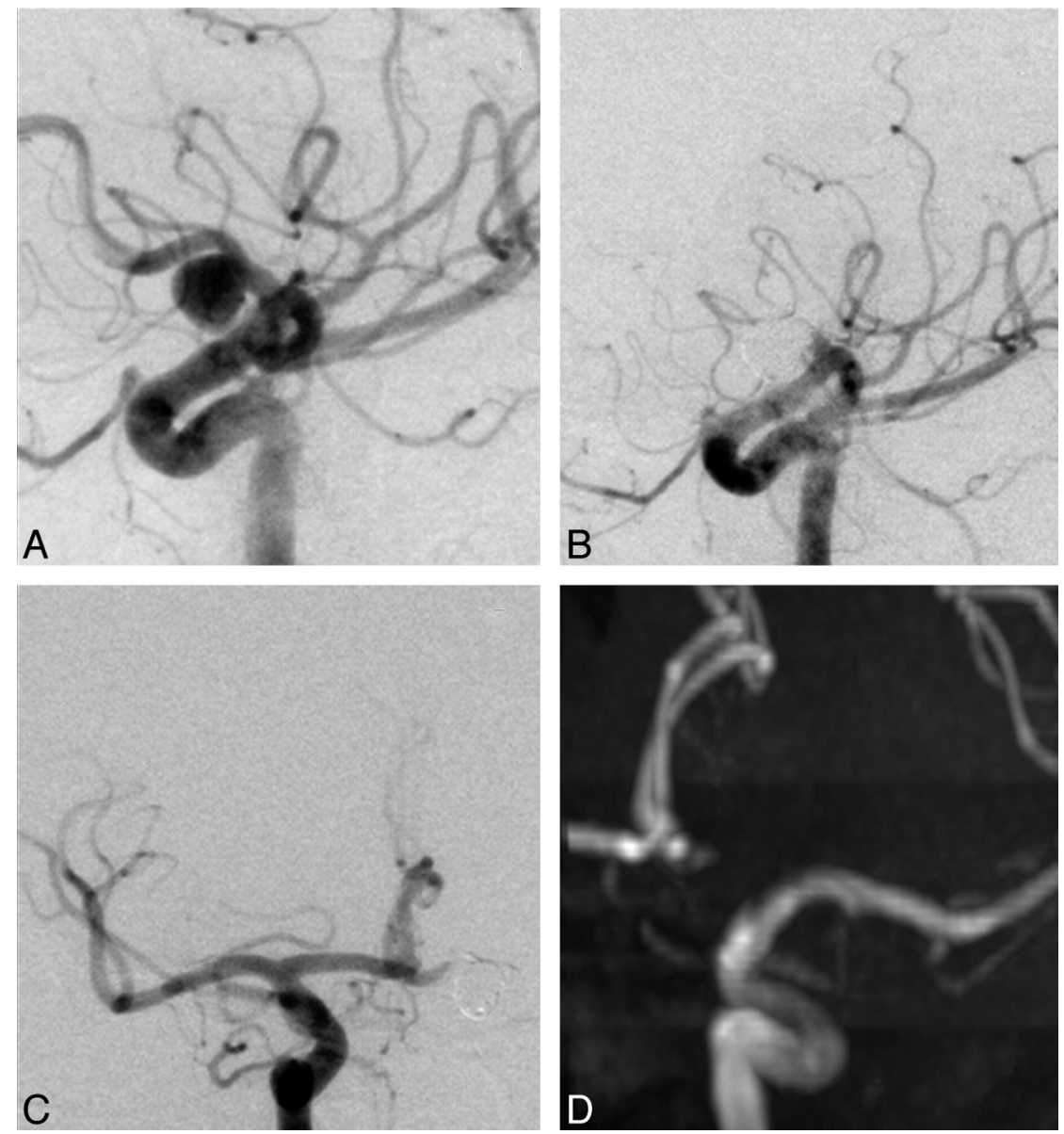

Fig 1. $A$ and $B$, Left carotid DSA oblique views show the aneurysm on the proximal third of the left $A 1$ segment $(A)$ and the result immediately after EVT (B). $C$, Right carotid DSA, anteroposterior view, after EVT shows the patency of the AcomA and contralateral A1 segment with filling of the distal left A1 segment. $D$, Maximum-intensity-projection reconstruction of the left carotid artery on an MRA 2.5 years after EVT

DSA at 6 months and 3 years revealed a recanalized A1 segment, without aneurysm recurrence.

\section{Results}

From January 2000 to January 2010, 401 ACA aneurysms (400 patients) were treated endovascularly with the following distribution: Twenty-four aneurysms were on A1 segments, 208 were at the A1-A2 junction, 119 were on the AcomA, and 50 were distal to the AcomA. Ruptured aneurysms accounted for $77 \%$ (308) of cases.

Of the $24 \mathrm{~A} 1$ aneurysms (24 patients), 18 (75\%) were located on the proximal third; $2(8 \%)$, on the middle third; and $4(16 \%)$, on the distal third of the segment. Eight (33\%) were treated acutely following SAH. Most (15/24) were treated by coiling without parent vessel occlusion. Those aneurysms were small (mean maximum diameter, $5.7 \mathrm{~mm}$ ) with a small neck (mean, $2.6 \mathrm{~mm}$ ), and none of them were recurrent aneurysms. Occlusion of the parent vessel was thought unnecessary for those cases. The other $9 \mathrm{~A} 1$ aneurysms were treated by coiling and parent vessel occlusion, and none, during the acute phase following SAH. These aneurysms were wide-neck with a maximum diameter ranging from 5 to $20 \mathrm{~mm}$ (mean, $11 \mathrm{~mm}$ ) and a neck size between 4 and $8 \mathrm{~mm}$ (mean, $5.3 \mathrm{~mm}$ ).

Clinical and imaging characteristics of patients and aneurysm are summarized in Table 1.

Of the 9 aneurysms treated by parent vessel occlusion, 5 were recurrences of previous treatments ( 3 patients had 1 session of EVT, 1 patient required 2 stages of EVT, and 1 patient had surgical clipping followed by endovascular treatment). One-third (3/9) of patients treated by parent vessel occlusion had an infarct on routine posttreatment CT; 1 patient (patient 8) was symptomatic (right hemiparesthesia). At the second follow-up visit (at 8 months), the symptoms had completely disappeared (Table 2).

Except for patient 8, who had a compression of her contralateral optic nerve due to her aneurysm, we did not observe any visual field defect at bedside clinical examination. Formal ophthalmology testing (ie, the Goldman Test) was, however, not performed systematically.

None of our patients or their families mentioned any emotional or intellectual disturbance after the EVT.

All aneurysms were followed up by imaging, ranging from 6 months to 9 years (median, 2.5 years). For the first control examination (5-10 months), 6 patients underwent DSA, with MRA for the remaining 3. Only 6 patients had long-term follow-up imaging (defined as imaging at least more than a year after treatment), generally by conventional angiography then followed by MRA. The latest examination used for determination of the degree of aneurysm occlusion consisted of 2 catheter angiograms and 4 MRAs.

Three patients had recanalization of the occluded A1 segment. Two were associated with a small recurrence at the neck 

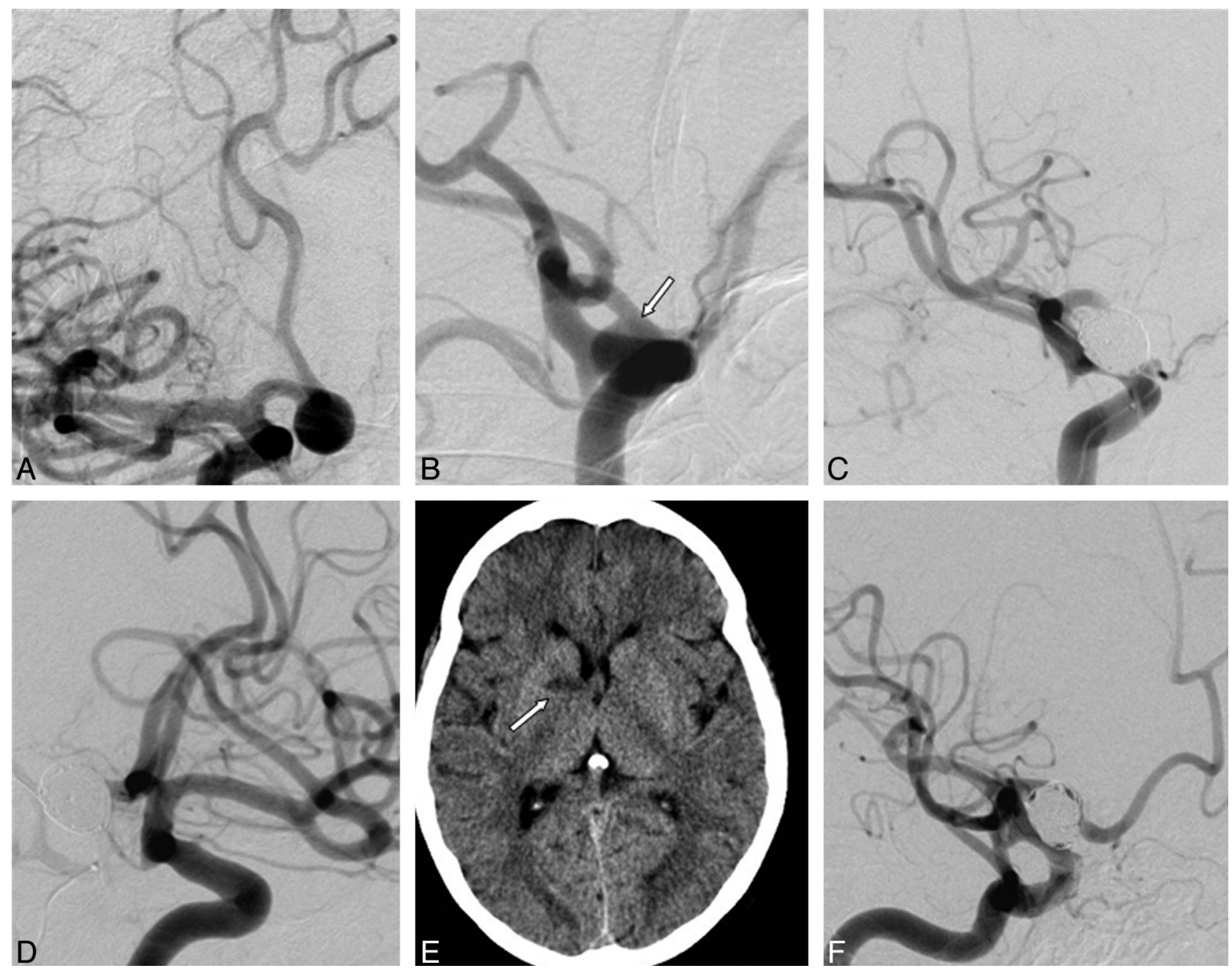

Fig 2. $A$ and $B$, Right carotid DSAs, anteroposterior view $(A)$ and oblique view $(B)$, show the aneurysm on the distal third of $A 1$ right segment (arrow). $C$, Right carotid DSA, oblique view, shows the immediate result after EVT. D, Left carotid DSA, oblique view, shows patency of the AcomA and contralateral A1 segment, with filling of the distal right A1 segment. $E$, Cerebral CT scan the day after EVT shows a stroke of the anterior pallidum and genu of the internal capsule (arrow). F, Control angiography at 3 years shows recanalization of the A1 segment without recurrence of the aneurysm.

\begin{tabular}{|c|c|c|c|c|c|}
\hline Case No. & Age $(y r) / S e x$ & Initial Presentation & Previous Treatment & Location & Size (N/MD) (mm) \\
\hline 1 & $56 / F$ & Previous SAH & 1 (c) & Mid (L) & $4 / 5$ \\
\hline 2 & $55 / F$ & Previous SAH & $2(s, c)$ & $\operatorname{Mid}(\mathrm{R})$ & $6 / 15$ \\
\hline 3 & $72 / \mathrm{M}$ & Previous SAH & 1 (c) & Prox (R) & $6 / 12$ \\
\hline 4 & $74 / F$ & Incidental & 0 & Prox (L) & $8 / 20$ \\
\hline 5 & $55 / F$ & Incidental & 0 & Prox (L) & $5 / 10$ \\
\hline 6 & $51 / F$ & Incidental & 0 & Dist (R) & $5 / 12$ \\
\hline 7 & $59 / F$ & Incidental & 0 & Dist (R) & $5 / 9$ \\
\hline 8 & $56 / F$ & Decreased visual acuity (OD) & 2 (c) & Dist (L) & $5 / 10$ \\
\hline 9 & $54 / F$ & Previous SAH & 1 (c) & Prox (R) & $4 / 6$ \\
\hline
\end{tabular}

of the aneurysm (Table 2). All recanalizations were diagnosed on the first follow-up DSA (at 6 months, 6 months, and 10 months, respectively), and both minor recurrences at the neck remained stable on long-term follow-up at 3 and 7 years, respectively. No patient had SAH during the period of follow-up.

\section{Discussion}

Proximal ACA (A1 segment) aneurysms are rare $(0.8 \%-3.4 \%$ of intracranial aneurysms $\mathrm{s}^{1-3}$. Suzuki et $\mathrm{al}^{1}$ retrospectively an- alyzed 4295 patients admitted for aneurysms in 3 neurosurgical centers between 1972 and 1990 and reported 38 (0.9\%) A1 aneurysms, including $37(37 / 38=97 \%)$ saccular, and 25 $(65.8 \%)$ proximal segment aneurysms.

\section{Treatment Options}

Treatment options include direct surgical clipping of the aneurysm neck, clip occlusion of the A1 segment, selective endovascular coiling with or without adjunct balloons or stents, endovascular parent vessel occlusion of the A1 as reported in 


\begin{tabular}{|c|c|c|c|c|c|}
\hline $\begin{array}{l}\text { Case } \\
\text { No. }\end{array}$ & EVT & $\begin{array}{c}\text { Infarct Post-EVT on } \\
\text { CT }\end{array}$ & Neurologic Deficit & $\begin{array}{l}\text { FU } \\
\text { (yr) }\end{array}$ & $\begin{array}{l}\text { Last Imaging } \\
\text { Findings }\end{array}$ \\
\hline 1 & $12 / 2000$ & No & 0 & 9 & Occlusion \\
\hline 2 & 03/2001 & No & 0 & 7 & $\begin{array}{l}\text { A1 recanalization, } \\
\text { small neck recurrence }\end{array}$ \\
\hline 3 & 07/2004 & No & 0 & 0.7 & Occlusion \\
\hline 4 & 09/2004 & Yes & 0 & 2.5 & Occlusion \\
\hline 5 & $10 / 2004$ & No & 0 & 2.5 & Occlusion \\
\hline 6 & $06 / 2005$ & No & 0 & 1.5 & $\begin{array}{l}\text { A1 recanalization, } \\
\text { aneurysm occluded }\end{array}$ \\
\hline 7 & $11 / 2006$ & Yes & 0 & 3 & $\begin{array}{l}\text { A1 recanalization, } \\
\text { small neck recurrence }\end{array}$ \\
\hline 8 & $01 / 2009$ & Yes & Right hemiparesthesia & 0.5 & Occlusion \\
\hline 9 & $06 / 2009$ & No & 0 & 0.5 & Occlusion \\
\hline
\end{tabular}

this series, and, more recently (but not yet reported in the literature), flow diverters.

Direct surgical clipping of the neck can be difficult. Hino et $\mathrm{al}^{3}$ reported, "Separating the perforating arteries from the neck or the dome of the Al aneurysms and preserving the vessel presents a substantial challenge to the surgeon, because the aneurysm is almost always behind the parent artery in the surgical field, making it difficult to achieve good access for this particular type of dissection."

Selective endovascular treatment of wide-neck aneurysms can be challenging and may require adjunct techniques. ${ }^{5,6}$ In the series of Lubicz et al, 5 of 8 patients with unruptured proximal A1 aneurysms were treated with balloon-assisted coiling. Stent-assisted coiling was reported for a large ruptured A1 aneurysm. ${ }^{8}$

The first 3 patients we treated with parent vessel occlusion had major recurrent aneurysms, ${ }^{4}$ which had previously been treated following SAH.

Specific comparisons of deliberate endovascular A1 parent vessel occlusion with other treatment options, in terms of complication and recurrence rates, is difficult, given the rarity of these aneurysms. The treatment remains a case-by-case decision in which individual expertise plays an important role.

\section{Ruptured versus Unruptured Aneurysms}

Considering that vasospasm may jeopardize collateral supply, it is theoretically better not to perform parent vessel occlusion during the acute phase of SAH. However, although we applied this rule for all our cases a priori, this principle remains untested in our study.

\section{Ischemia Related to A1 Occlusion}

Angiographic demonstration of bilateral A1s and a patent AcomA by contralateral common carotid compression are very likely adequate tests to predict sufficient blood flow to prevent ischemia. ${ }^{9}$ In cases in which the treating neuroradiologist was unsure, a temporary balloon occlusion test was performed. The occlusion of perforators originating from the A1 segment, nonetheless, remains a concern.

According to Perlmutter and Rhoton, ${ }^{10}$ in an anatomic study of 100 hemispheres, 2-15 perforating arteries arose from the A1 segment. The majority $(68 \%)$ came from the lateral portion of the A1 segment. They terminated in the anterior perforated substance (41\%), the dorsal surface of the optic chiasm or the suprachiasmatic portion of the hypothalamus
(29\%), the optic tract (11\%), the dorsal surface of the optic nerve $(2 \%)$, between the cerebral hemispheres $(2 \%)$, and on the inferior surface of frontal lobes $(10 \%)$. The A1 perforators constantly supply the optic chiasm and the anterior part of margins of the hypothalamus and third ventricle area. Occasionally, Al perforator arteries may also vascularize the caudate nucleus and globus pallidus. ${ }^{10}$

The largest perforating branch of the ACAs is the RAH. The recent anatomic study of Avci et $\mathrm{al}^{11}$ on 62 hemispheres showed that the RAH was unilateral in $75 \%$ of cases and most often arose from the communicating segment $(28.5 \%)$ or the A2 segment (63.6\%) and rarely from the A1 segment (7.8\%). The RAH vascularizes the anterior limb of the internal capsule, the head of caudate nucleus, and the anterior lentiform nucleus. ${ }^{10}$ A1 aneurysms usually arise at the point of origin of a minor perforating artery, not at the origins of cortical branches or the RAH. ${ }^{1,12,13}$ Occlusion of the RAH may cause upper extremity weakness; palsies of the face, palate and tongue; and sometimes aphasia (in the dominant hemisphere). ${ }^{10}$

When A1 perforators are occluded, typical symptoms due to infarcts in the anterior hypothalamic region include emotional changes, personality disorders, and intellectual deficits. $^{2,14,15}$ To limit the risk of perforator infarction, it may be safer to carefully look for the RAH and to limit the length of the coil occlusion to the segment of the parent vessel that faces the neck of the aneurysm. We may have missed some asymptomatic infarcts that would have appeared on MR imaging. Furthermore, we did not determine neuropsychological status at baseline or following endovascular occlusion, and some subtle changes may have been missed.

\section{Recanalization of the A1 Segment and Recurrence of Aneurysm}

Recanalization of the A1 segment occurred in one-third of patients, always on the first follow-up angiogram. Three patients were followed up with MRA only, which may have led to an underestimation of small recurrences. Ipsilateral injection of the recanalized A1 on control DSA showed opacification of the AcomA and contralateral A1, which was in favor of a true lumen recanalization. Unfortunately, contralateral injections were not available on all control DSAs. Recanalization of vessels occluded with coils has often been documented, for example, at the level of the vertebral ${ }^{16,17}$ and posterior cerebral arteries. ${ }^{18}$ Despite the possibility of recanalization of the A1 
segment and the higher risk of recurrence in wide-neck aneurysms, ${ }^{8}$ none of our patients had a major recurrence, including the 6 patients with long-term follow-up ( $\geq 18$ months).

\section{Conclusions}

In this series, parent artery occlusion was effective in treating wide-neck aneurysms arising from the A1 segment of the ACA in patients with adequate collateral supply.

\section{References}

1. Suzuki M, Onuma T, Sakurai Y, et al. Aneurysms arising from the proximal (A1) segment of the anterior cerebral artery: a study of 38 cases. J Neurosurg 1992;76:455-58

2. Aoki Y, Nemoto M, Yokota K, et al. Ruptured fusiform aneurysm of the proximal anterior cerebral artery (A1 segment). Neurol Med Chir (Tokyo) 2007;47:351-55

3. Hino A, Fujimoto M, Iwamoto Y, et al. Surgery of proximal anterior cerebral artery aneurysms. Acta Neurochir (Wien) 2002;144:1291-96

4. Raymond J, Guilbert F, Weill A, et al. Long-term angiographic recurrences after selective endovascular treatment of aneurysms with detachable coils. Stroke 2003;34:1398-403

5. Viñuela F, Duckwiler G, Mawad M. Guglielmi detachable coil embolization of acute intracranial aneurysm: perioperative anatomical and clinical outcome in 403 patients. 1997. J Neurosurg 2008;108:832-39

6. Debrun GM, Aletich VA, Kehrli P, et al. Aneurysm geometry: an important criterion in selecting patients for Guglielmi detachable coiling. Neurol Med Chir (Tokyo) 1998;38(suppl):1-20

7. Lubicz B, Bruneau M, Dewindt A, et al. Endovascular treatment of proximal anterior cerebral artery aneurysms. Neuroradiology 2009;51:99-102
8. Gupta R, Horowitz MN, Gilman S. Neuroform stent-assisted coil embolization of a ruptured A1 segment anterior cerebral artery aneurysm. J Neuroimaging 2006;16:117-19

9. Hetzel A, von Reutern G, Wernz MG, et al. The carotid compression test for therapeutic occlusion of the internal carotid artery: comparison of angiography with transcranial Doppler sonography. Cerebrovasc Dis 2000;10:194-99

10. Perlmutter D, Rhoton AL Jr. Microsurgical anatomy of the anterior cerebralanterior communicating-recurrent artery complex. J Neurosurg 1976;45: 259-72

11. Avci E, Fossett D, Aslan M, et al. Branches of the anterior cerebral artery near the anterior communicating artery complex: an anatomic study and surgical perspective. Neurol Med Chir (Tokyo) 2003;43:329-33

12. Handa J, Nakasu Y, Matsuda M, et al Aneurysms of the proximal anterior cerebral artery. Surg Neurol 1984;22:486-90

13. Wakabayashi T, Tamaki N, Yamashita H, et al. Angiographic classification of aneurysms of the horizontal segment of the anterior cerebral artery. Surg Neurol 1985;24:31-34

14. Riggs HE, Rupp C. Variation in form of circle of Willis: the relation of the variations to collateral circulation-anatomic analysis. Arch Neurol $1963 ; 8: 8-14$

15. Ostrowski AZ, Webster JE, Gurdjian S. A study of the proximal portion of the anterior cerebral artery: the areas of the brain it supplies. Surg Forum 1960;10:788-90

16. Baik SK, Kim YS, Lee HJ, et al. Antegrade recanalization of parent artery in internal trapping of vertebral artery dissecting aneurysm: a case report. Surg Neurol 2007;68:108-11

17. Sawada M, Kaku Y, Yoshimura S, et al. Antegrade recanalization of a completely embolized vertebral artery after endovascular treatment of a ruptured intracranial dissecting aneurysm: report of two cases. J Neurosurg 2005;102:161-66

18. Horie N, Kawahara I, Kitagawa N, et al. Recanalization after endovascular occlusion of a dissecting aneurysm of the posterior cerebral artery: a case report and review of the literature. Clin Neurol Neurosurg 2008;110:411-15 